\title{
El idioma como pauta de intercambio. Tributo a Georg Simmel
}

\section{Amado Alarcón}

Universitat Rovira i Virgili. Departament de Gestió d'Empreses Av. Universitat, 1. 43204 Reus (Tarragona). Spain amaa@fcee.urv.es

\section{Resumen}

Los desarrollos teóricos de Simmel permiten, a través de la idea de intercambio, establecer un puente entre las dimensiones micro y macro en sociología. A través del concepto de intercambio podemos examinar la emergencia de pautas de interacción como idiomas y dinero, así como la relación entre éstas y sus consecuencias sobre las decisiones de los individuos.

Palabras clave: intercambio, dinero, idioma, círculo económico, cierre social.

\section{Abstract. The language as a model of interchange. Tribute to Georg Simmel}

Exchange in Simmel's theoretical works operates as a bridge between micro and macro levels of analysis in sociology. This concept allows to study the emergence of interaction forms as language and money, their reciprocal influences and the consecuences to individuals.

Key words: exchange, money, language, economical circle, social clousure.

\section{Sumario}

\section{Introducción}

2. Idiomas como pauta de intercambio

3. La valoración económica de los idiomas y extensión del círculo económico
4. El círculo económico y el cierre social 5. Conclusiones

Bibliografía

\section{Introducción}

Para Simmel, la «sociología» es una ciencia dedicada al estudio de «interacciones» entre individuos ${ }^{1}$. El trabajo sociológico, desde esta perspectiva, consiste en el estudio de las interacciones como puente entre los individuos y las

1. Weber comenta los trabajos de Simmel indicando la ambigüedad del término, matizando que como influencia recíproca de varias 'unidades' se dan 'interacciones' (Weber, 1981: 150). 
formas de cultura objetiva y otros rasgos estructurales, como el tamaño de las comunidades de interacción. La interacción se produce por medio de diferentes formas o pautas de interacción, unas históricamente más desarrolladas que otras. Así, siguiendo a Simmel, en sociedades primitivas tendríamos formas extendidas de interacción como el «robo»y el «regalo». Pero, posiblemente, la forma de interacción de mayor interés para el sociólogo en el mundo actual sea la del «intercambio» ${ }^{2}$, puesto que alrededor de los intercambios emergen formas culturales objetivas como el dinero y el idioma, que son consecuencia del carácter social del hombre pero distintas a los individuos particulares ${ }^{3}$.

Como indica Ritzer, para Simmel las pautas de interacción constituyen la esencia de las estructuras sociales (Ritzer, 1995: 331). Existe un mecanismo por el que los intereses de los individuos se satisfacen con el intercambio. Las formas sociales de intercambio se generan y se estandarizan a fin de proporcionar un patrón estable en la provisión de beneficios para los individuos. Estas formas sociales de intercambio son en sí formas diferentes a los sujetos, constituyen "hechos sociales» que tienen influencia sobre otros «hechos sociales», como el tamaño del grupo o la extensión de ciertas características entre el conjunto de los individuos, como la individualización, la diferenciación o propensión a la valoración económica de todos los aspectos de la vida, generándose así nuevas necesidades individuales que determinen las formas más adecuadas para los intercambios. La relación entre sujetos de dar y recibir en las interacciones, lejos de la mera reciprocidad tendiente a un equilibrio contingente entre individuos, se circunscribe a unas pautas, formas o normas de intercambio. Las pautas de interacción son previas a los agentes que participan, si bien se originan en los sujetos y en las relaciones entre éstos, así como se hallan sujetas a mantenimiento, transformación o supresión a partir de la acción de los propios agentes, si bien la dificultad para alterar las formas que adopta la cultura objetiva se hace mayor a medida que ésta se consolida.

2. «[...] así se ha intentado definir al hombre como una animal político, el animal que fabrica herramientas, el animal que determina sus objetivos, el animal jerárquico, y hasta, por parte de un filósofo serio, como el único que es víctima de megalomanía. Quizá se pueda añadir a esta serie la definición de que el hombre es el animal que intercambia; y esto no es en realidad más que un lado o forma de la característica más general en la que parece consistir lo específico de los seres humanos, esto es, el ser humano es el animal objetivo. En ninguna otra parte del mundo encontraremos siquiera sean vestigios de lo que llamamos objetividad, la observación y manipulación de las cosas que se sitúan más allá del sentimiento y la voluntad subjetivos» (Simmel, 1977: 346-347).

3. Considero, como Homans, Blau, Olson o Coleman, que la interacción supone intercambio de bienes materiales y no materiales. 


\section{Idiomas como pauta de intercambio ${ }^{4}$}

Los idiomas constituyen una pauta o norma de intercambio universal. Sin embargo, hasta el presente, la universalidad del medio, intrínseco a la naturaleza social del hombre, se ha compaginado con una diversidad idiomática que posibilita el intercambio para los más diversos fines en el seno de múltiples comunidades lingüísticas no necesariamente exclusivas ${ }^{5}$. La diversidad lingüística se evidencia al observar las líneas evolutivas divergentes de los idiomas en grupos voluntaria o involuntariamente cerrados al intercambio, hasta el punto que en el presente coexisten unos seis mil idiomas en el mun$\mathrm{do}^{6}$. Pese a la forma aparentemente gratuita en que se administra a los sujetos el idioma en las sociedades occidentales, la capacidad de capitalización lingüística ${ }^{7}$ de los individuos es altamente limitada si la comparamos con el número de idiomas existentes. Ello indica que podemos considerar los idiomas como un recurso escaso desde el punto de vista de capitalización. Por otra parte, si por algún motivo los individuos encuentran necesidad de relacionarse con otros con los que inicialmente no comparten un determinado repertorio lingüístico, y si tienen suficientes incentivos para ello, aparecerá la necesidad entre éstos de adoptar unas determinadas pautas o normas de interacción, siendo una - sino la principal_ el idioma. Probablemente, a mayor tamaño de la comunidad, mayor diferenciación y mayor dependencia como consecuencia de la división del trabajo entre los miembros de la comunidad, mayor será la necesidad de establecer unas pautas lingüísticas que regulen las necesidades comunicativas orientadas a fines diversos.

4. Si la lengua no fuera más que una herramienta neutral e intercambiable de comunicación (siendo la comunicación, además, simple transmisión de información), no se entendería la pasión que despierta el problema lingüístico. Cabe recordar que incluso los autores del 'método económico' indican que la comunicación es sólo uno de los propósitos del lenguaje, entre los que se encuentran la identidad/solidaridad y el control/dominación. Si la lengua fuera un mero instrumento de comunicación, difícilmente generaría los debates periodísticos y políticos que en la actualidad suscita (Pool, 1991).

5. «Por una comunidad lingüística se entiende aquélla cuyos miembros participan por lo menos de una variedad lingüística y de las normas para su uso adecuado» (Fishman, J. 1979: 54).

6. El número de idiomas se está reduciendo notablemente en la actualidad.

7. Podemos considerar el capital humano como el estoc de habilidades, salud mental y física, conocimientos y todo aquello que contribuye a incrementar los rendimientos de una persona en el momento presente y en el futuro. El incremento de capital humano es el resultado de la inversión de recursos en formación. Entre los componentes del conocimiento y por tanto del capital humano están los lenguajes: tanto la primera lengua o lengua materna como el resto. Las habilidades lingüísticas satisfacen los tres componentes básicos del capital humano: $a$ ) incorporados en la persona; $b$ ) productivos en el mercado de trabajo; c) se crean sacrificando tiempo y otros recursos (Chiswick \& Miller, 1995). Desde este punto de vista, aprender una lengua, ya sea perfeccionar la propia o adquirir conocimientos en otra, supone una inversión de recursos, por ejemplo, en la compra de diccionarios, clases, libros o tiempo no destinado a otras actividades. 
¿Cómo surgen los idiomas?, o más importante para muchos individuos en nuestra sociedad: ¿cómo se mantienen, adquieren hegemonía o desaparecen los idiomas (o más propiamente los hablantes de éstos)? Dado que hipotéticamente la emergencia de las pautas de intercambio, al menos en su origen, obedece a intereses individuales o corporativos ${ }^{8}$, ya sea en contextos cooperativos o conflictivos, cabría explicar el mantenimiento o la supresión de la diversidad idiomática (en particular, las tendencias hacia la homogeneización lingüística, así como la desaparición de lenguas minoritarias) en relación con los procesos de integración monetaria. Más concretamente, como se mantienen idiomas diferentes - o ausencia de un repertorio común - en el seno de comunidades monetarias más amplias. En este sentido, las características particulares de los idiomas, y el modo en que éstos han sido históricamente dados/facilitados a los individuos, tiene unas pautas análogas a las del dinero 9 . Entre las más destacadas se encuentran, por una parte, que la provisión del idioma oficial como la de la moneda «oficial» corre a cargo de los gobiernos y, por otra, la persecución histórica que por parte de los gobiernos se ha realizado hacia formas alternativas de idioma o moneda diferentes a la oficial. Sin embargo, los estadios de integración presente de las comunidades monetarias e idiomáticas no son en absoluto idénticas.

Dado que consideramos los idiomas como pautas de interacción, cabe indicar que procedimientos regulativos, basados en los intereses individuales o corporativos, operan en el establecimiento de los mismos. Históricamente se crean normas, formas o pautas sobre como intercambiar en beneficio y perjuicio de algunos o de todos. Podríamos aducir que «la manifestación más clara de la necesidad de normas sociales se encuentra en las situaciones sociales en las que los intereses de todas las partes, no sólo de la mayoría de ellas, requiere una protección por parte de las normas sociales, porque la búsqueda del propio interés sin limitaciones normativas perjudica los intereses de las partes implicadas». Por otra parte, el establecimiento de determinadas normas de intercambio entre sujetos particulares de un todo ecológico, esto es, que pueden afectar a otros sujetos, puede conducir también a la necesidad de normas. A partir del ejemplo del dilema del pri sionero, Blau concluye que las normas sociales que prohiben las acciones por medio de las cuales los individuos pueden obtener ventajas a expensas de los intereses comunes de la colectividad son necesarias (Blau, 1982: 215-216). Podemos considerar estas normas de intercambio como bienes públicos que han de ser provistos por medio de alguna acción colectiva, condicionada por

8. Dada la importancia en nuestros días de este tipo de actores, todo análisis que subestimara la capacidad de acción de, por ejemplo, gobiernos y empresas multinacionales, perdería una inmensa capacidad heurística.

9. Entre las más destacadas se encuentran la provisión del idioma oficial, como la de la moneda «oficial» corre a cargo de los gobiernos y la persecución histórica por parte de los gobiernos de formas alternativas de idioma o moneda distinta a la oficial. 
factores como el tamaño del grupo ${ }^{10}$, la existencia de grupos privilegiados o incentivos selectivos (Olson: 1992). Por otra parte, emergencia que, si se produce y en la forma que adopta, no es necesariamente intencionada ${ }^{11}$. Podemos considerar tanto la aparición como la supresión o la modificación de las normas de intercambio como una consecuencia del interés de los sujetos sobre el control sobre las externalidades de la acción (Coleman: 1990: 249-259). De hecho, las constituciones siempre imponen barreras al intercambio que separan los derechos políticos y los recursos de carácter público (en la forma de derechos de legislativos, ejecutivos o ciudadanos para participar en las decisiones colectivas) de otros recursos, particularmente de aquéllos de los sectores privados de la economía. Estas barreras nunca son absolutas y siempre permiten intercambio entre el control de las decisiones colectivas y el control de otros recursos, particularmente los recursos económicos (Coleman, 1990: 847). Cuando las acciones de cada uno tienen efectos (externalidadades) para los demás en un sistema, la creación de un contrato social en el que cada uno otorga derechos de control sobre sus acciones a los demás constituye una solución al problema hobbesiano. La barrera es una realidad política relativa a la distribución de costes y beneficios $^{12}$. Dadas estas consideraciones acerca de la regulación de los intercambio, ¿qué papel pueden jugar los idiomas en la limitación estructural de los intercambios? La respuesta que se aportará es que los idiomas emergen del control e interés que los individuos presentan sobre bienes materiales o

10. Como bien público opcional en un contexto multilingüe, tanto Laitin (1988) como Grin (1990) ya han indicado que las estructuras lingüísticas (número de hablantes, recompensas ligadas al uso de una lengua...) son determinantes de las estrategias de elección individual $y$, en consecuencia, del uso y la supervivencia de las lenguas.

11. «Mientras que la concepción de la reciprocidad en el intercambio implica la existencia de fuerzas equilibradoras que crean una tensión hacia el equilibrio, las operaciones simultáneas de diversas fuerzas equilibradoras producen una fuerza recurrente de desequilibrios en la vida social y la dialéctica resultante entre reprocidad y el desequilibrio da a las estructuras sociales su naturaleza y su dinámica distintivas.» (Blau, 1982: 11)

12. Los conceptos básicos del micronivel de análisis son: $a$ ) actores $-i-; b$ ) recursos $-j-$. Las relaciones entre los elementos son: $a$ ) interés del actor i en el recurso $j-X i j-; b$ ) Control del actor i sobre el recurso $j-C i j-$. Siendo $X i j$ relativo al interés de $i$ en todos los $j$ del sistema; mientras que la proporción de $C i j$ depende de la cantidad de $j$ en el sistema. Los conceptos del macronivel de análsis son: $a$ ) El valor del recuso $j-V j-$ es la suma del interés de los actores en el recurso una vez ponderado el interés de cada actor según su poder; b) el poder de un individuo - $R i$ - es un concepto del nivel-sistema. El poder de un individuo es la suma del valor de los recursos que éste controla $-R i=C i j V j-$. De esta forma, el control de recursos en los que otro está interesado constituye poder. La relación entre dos bienes es el ratio de intercambio de un bien por otro. Así, el valor y el poder dependen de la estructura de intereses y control, así como de una serie de supuestos sobre los procesos sociales por los que el intercambio tiene lugar. Valor y poder pueden ser considerados como resultado de las acciones de una constelación particular de actores, con unos intereses particulares y unos recursos, donde cada uno pretende maximizar la utilidad sujeto a las limitaciones del control inicial. 
inmateriales que se hallan en su entorno ${ }^{13}$, definiendo comunidades y formas de intercambio acordes con sus intereses ${ }^{14}$. Veamos primero qué tipos de consideraciones económicas recaen sobre los idiomas para posteriormente observar la regulación que se crea en torno a los mismos.

\section{La valoración económica de los idiomas y extensión del círculo económico}

Tal y como indica Simmel, dado un determinado orden social en que los individuos poseen una tendencia progresiva hacia la actitud económica ante todos los aspectos de la vida ${ }^{15}$, encontramos rasgos estructurales que perfilan claramente el valor que los sujetos pueden conferir a los idiomas, a fin de mejorar su situación en los intercambios económicos. La propiedad de los recursos lingüísticos en forma de capital lingüístico elimina a la vez que crea la barrera al intercambio y al disfrute de los recursos, de la misma forma que un patrón monetario único sitúa en un plano de igualdad a los sujetos en el intercambio. La extensión del círculo económico-monetario presenta ciertas analogías con la extensión del "círculo lingüístico». Así, en cuanto al primero, Simmel indica que «la general aceptación de dinero [...] hace que su acción se extienda indefinidamente y convierta a todo el mundo civilizado en un único círculo económico de intereses recíprocos, producciones que se complementan y usos análogos. Por otra parte, el dinero produce una enorme individualización del hombre económico. [...] Las normas liberales, ligadas a la economía monetaria, ponen al individuo en competencia libre con los demás, y, finalmente, aquella competencia y aquella extensión del círculo económico, determinan una especialización de las actividades y una explotación total de los talentos especiales, que sólo es posible por las compensa-

13. Aquí, el término entorno, siguiendo a Simmel, indica una relación de distancia tal con los bienes que no haga de éstos algo inalcanzable o demasiado próximo o abundante, y por la tanto no sujeto a valoración económica.

14. El enfoque de ecosistemas comunicativos (Laponce, 1985; 1993) ilustra la relación entre lengua y territorios como espacios de alta densidad en los intercambios de comunicación (territorios siempre determinados por tecnologías, p.e. un puente, el ferrocarril o Internet). Por otra parte, si la satisfacción de un individuo se produce mediante el control de bienes de los que éeste no dispone en suficiente cantidad, y dichos bienes se encuentran en disposición de otros actores, deberá establecer un intercambio con dichos actores. Para ello deberá compartir el medio lingüístico de la interacción.

15. «En gran medida [...] la economía monetaria y las tendencias liberales, unidas a ella, aflojaron [...] las asociaciones estrechas, desde las gremiales a las nacionales, e inauguraron la economía mundial. Pero, por otra parte, favorecieron el desarrollo de un egoísmo económico sin escrúpulos. Cuanto menos conoce el productor al consumidor, por obra de la extensión del círculo económico, tanto más exclusivamente se concentra su interés en la altura del precio que puede obtener de él. Cuanto más impersonal y desprovisto de cualidades aparece ante él su público, tanto más responderá a la consecución exclusiva del resultado, puramente cuantitativo, del trabajo: el dinero.» (Simmel: 1986: 792) 
ciones que pueden surgir en el marco de un círculo muy grande. Dentro de la economía, el dinero es el lazo que pone en relación la extensión máxima del grupo económico y la diferenciación máxima de sus miembros, en el sentido de la libertad y de la autonomía, como de la diferenciación cualitativa del trabajo. $\mathrm{O}$, dicho más exactamente, gracias al dinero, el grupo de la economía natural, pequeño, cerrado y uniforme, se convierte en otro, cuyo carácter unitario se escinde en los dos aspectos de la ampliación y la individualización» (Simmel: 1986: 779-80).

En cuanto a la extensión del "círculo lingüístico», los beneficios colectivos de la homogeneización lingüística, en perspectiva económica, según Gellner, se derivan de que ésta contribuye y habilita el progreso económico de la sociedad - la sociedad del perpetuo crecimiento - y la movilidad social del individuo. Particularmente, el papel del Estado, que contribuye a la educación homogénea hace posible una masa laboral intercambiable laboral y geográficamente en el territorio homogeneizado lingüísticamente ${ }^{16}$. Un estado, un idioma, parece haber sido la consigna del estado "científico». Dicho estado es, según Smith, "una politeya que intenta homogeneizar a la población situada dentro de sus confines administrativos, utilizando las técnicas y los métodos científicos más avanzados en aras de la eficiencia. Los gobernantes usan la máquina burocrática y los frutos de la investigación científica y de las aplicaciones tecnológicas con objeto de procurarse recursos y movilizar a la población de su territorio", hasta el punto que el deber patriótico del nacio-

16. El Estado, una institución especializada y estable que mantiene el orden —en referencia a la definición weberiana del mismo-, nace en la etapa agraria como opción. En cambio, en la sociedad industrial el Estado es «ineludible» en la medida en que "las sociedades industriales son extraordinariamente grandes y, para mantener el nivel de vida al que se han acostumbrado (o desean habituarse fervientemente), dependen de una división general del trabajo y la cooperación increíblemente compleja» donde la cooperación debe ser un bien a proteger (Gellner, 1988: 17-18). Ello se debe a que las necesidades lingüísticas de una sociedad a otra varían, dado que «en el orden social tradicional, los lenguajes de la caza, de la agricultura, de rituales diversos, de la asamblea, de la cocina o del harén, forman sistema autónomos [...] En cambio, en nuestra sociedad se da por supuesto que todos los usos referenciales del lenguaje remiten en última instancia a un mundo coherente y se pueden reducir a un idioma unitario" (Gellner, 1988: 38). Así, la homogeneización lingüística no es un objetivo de las autoridades políticas en las épocas preindustriales si tenemos en cuenta el objetivo arriba indicado. En la sociedad estamental «la alfabetización, el establecimiento de una escritura hasta cierto punto permanente y normalizada, significa la posibilidad real de llevar a cabo un acopio y una centralización de la cultura y el conocimiento». Sin embargo, "el estrato dirigente [...] hace hincapié, más que en la homogeneidad, en la diferenciación cultural». La construcción de la nación es un requisito para la extensión del sistema industrial, es un elemento estructural. La conexión, según Gellner, es la que sigue: la sociedad industrial precisa de la homogeneización de los individuos para su intercambiabilidad o movilidad social. Dicha homogeneización administra una base cultural homogénea o (re)construye una nación. La homogeneización se lleva a cabo por el «Estado científico» como administrador del progreso. En este sentido, «el nacionalismo no hace más que reflejar la necesidad objetiva de homogeneidad» (Gellner, 1988: 67). 
nalista es el crecimiento económico de su nación (Smith, 1976: 320) ${ }^{17}$. Estas consideraciones no nos han de alejar de los microfundamentos de la emergencia de idiomas de intercambio. Las consideraciones arriba indicadas parten de intereses concretos en la parte empresarial y gubernamental en términos de beneficios derivados de la inversión en capital lingüístico, dando lugar, sobre todo para el último actor indicado, la categoría de actor privilegiado. El Estado pretende la administración y la defensa de un idioma único a nivel territorial, en la medida en que le permite la administración eficiente de la sociedad (Friedman, 1977). Laitin ha centrado su atención bajo una perspectiva histórica y de elección racional en la importancia de la lengua como elemento estratégico de los gobernantes, con el objetivo de conseguir una administración ordenada y eficiente de sus territorios, maximizando la extracción de recursos en sus confines territoriales, siendo ésta una aproximación que escapa al espacio histórico de la sociedad industrial ${ }^{18}$.

Respecto al alcance (número de sujetos afectados) de la norma de intercambio lingüística, podemos microfundamentar una de las características macro que caracterizan a los idiomas, esto es, de la tendencia de un sólo idioma a operar como monopolio, a partir de las decisiones individuales. Se trata de una analogía de idioma como moneda que indica que las lenguas con un mayor potencial comunicativo tienden a operar como monopolios, de la misma manera que en el comercio internacional resulta más eficiente el uso del oro primero y después el dólar (Carr, 1985). Ello se debe a que las lenguas con un mayor potencial comunicativo tienden a generar externalidades de red, es decir, la extensión de la red lingüística de inmediato genera economías de escala (Church \& King, 1993) o una forma de efecto "furgón de cola». Así, en la medida que los individuos no son excluibles de su suministro, podemos indicar que los idiomas son bienes hipercolectivos, es decir, además de cumplir con las condiciones de bien colectivo, los beneficios individuales aumentan con la incorporación de nuevos consumidores. Así, la utilidad, considerada esta como el potencial comunicativo que proporciona un idioma, para los individuos se incrementará conforme aumenta el número de hablantes de un idioma en el momento $t_{0+1}$ (De Swaan, 1998: 68-72).

17. La historia económica de nuestra sociedad parece unida al mantenimiento de la sociedad del «perpetuo crecimiento» y es debido a la creación del Estado moderno como instrumento de gobierno que precisa sus propias fuentes de legitimidad. Una vez administrada una base homogénea de cultura sobre la población de un Estado — constituida por tanto la base del Estado nación, "para el nacionalista, el desarrollo económico es una cuestión de orgullo y deber patriótico. La prosperidad y, más aún, los signos de prosperidad son marcas de realización colectiva" (Tivey, 1987: 90). Es por ello que, en la medida en que se desarrolla la sociedad industrial, es preciso que se establezcan las bases de la economía de base industrial y una forma de organización del capital.

18. Este enfoque ha sido desarrollado teóricamente por Laitin, aplicando el modelo a diversos estudios de casos. Véase Laitin (1989, 1992), Laitin \& Rodríguez (1992), Laitin \& Solé \& Kalavitas (1994) para un examen del caso catalán. 


\section{El círculo económico y el cierre social}

Sólo se puede generar igualdad entre aquéllos que comparten el patrón monetario, del mismo modo que sólo se posibilita el intercambio libre con un idioma común. La decisión - generalmente política, siempre social— de definir una comunidad monetaria o lingüística atiende a una decisión estratégica sobre el tamaño de la comunidad, del círculo de intercambios. Frente a la tendencia histórica del aumento del círculo económico como consecuencia intrínseca de los beneficios a escala de los intercambios, el cierre social ${ }^{19}$ es una de las formas de organización social del intercambio económico. Para indicar el significado de cierre social podemos partir de la idea de relación social «abierta» $\mathrm{y}$ «cerrada» de Weber: «Una relación social (lo mismo si es de "comunidad» como de «sociedad») se llama «abierta» al exterior cuando y en la medida en que la participación en la acción social recíproca que, según su sentido, la constituye, no se encuentra negada por los ordenamientos que rigen esta relación a nadie que lo pretenda y esté en situación real de poder tomar parte en ella. Por el contrario, llámese «cerrada» al exterior cuando y en la medida en que aquella participación quede excluida, limitada o sometida a condiciones por el sentido de la acción o por los ordenamientos que la rigen [...] El cierre de tipo racional se basa especialmente en la siguiente situación de hecho: una relación social puede proporcionar a sus partícipes determinadas probabilidades de satisfacer ciertos intereses, tanto exteriores como interiores, sea por el fin o por el resultado [...] Cuando los partícipes de esa relación esperan que su propagación les ha de aportar una mejora de sus probabilidades en cantidad, calidad, seguridad o valor, les interesa su carácter abierto; pero cuando, al contrario, esperan obtener esas ventajas de su monopolización, les interesa su carácter cerrado al exterior» (Weber, 1993: 35).

Para Weber, la elección del idioma como criterio para el cierre social no es puramente una cuestión del papel en el intercambio social, en cierta forma es un elemento arbitrario de segmentación: «El número creciente de los que compiten en comparación con las posibilidades adquisitivas acrecienta el interés de los partícipes en limitar su número de algún modo. La forma en que esto suele ocurrir es que se tome algún carácter exteriormente comprobable de los competidores (reales o potenciales), por ejemplo: la raza, el idio-

19. El concepto weberiano de cierre social «consiste en el proceso mediante el cual las actividades sociales buscan ampliar al máximo sus recompensas, limitando el acceso a los recursos y a las oportunidades a un número restringido de candidatos. De esta forma, los grupos cerrados monopolizan determinadas oportunidades para sus miembros, restringiendo a los ajenos al grupo el acceso a los beneficios de tales oportunidades [...] Un grupo es cerrado cuando presenta las siguientes condiciones: $a$ ) una frontera fuerte entre la concepción del «nosotros» y el «ellos»; b) una alta densidad en términos reticulares, que proporciona control sobre la entrada de nuevos miembros; $c$ ) exclusión de los sujetos extraños con la intención de asegurar para el grupo una posición privilegiada a expensas de otros; (d) un conjunto de normas y valores relativamente rigurosos que obliga a los miembros a mantener una determinada conducta» (Requena, 1998: 339). 
ma, la religión, el lugar de nacimiento, la clase social, el domicilio, etc., para basar sobre él la exclusión. Es indiferente, en los casos concretos, el carácter que se escoja para la eliminación; puede echarse mano del primero que se encuentre»(Weber, 1993[1922]: 276). Sin embargo, los idiomas, y particularmente los minoritarios, en la medida que definen comunidades bien delimitadas y proximidad entre sus miembros, pueden constituir una excelente base para impedir el alejamiento de los recursos de los individuos que se produce en el intercambio. Aquellos recursos a los que el individuo está dispuesto a renunciar en el intercambio pueden permanecer en una comunidad relativamente próxima que permita un fácil acceso posterior en la recuperación de los mismos.

La formación de una norma de intercambio en base al idioma, con frecuencia, se trata de una construcción colectiva, con resultado normativo ${ }^{20}$, de un bien jurídico. Siguiendo a Weber, «la totalidad de los que concurren en común se convierte, respecto al exterior, en una "comunidad de interesados», sin perjuicio de la competencia entre sí; crece la tendencia a que «se origine algún tipo de sociedad» —-socialización— racionalmente organizada y, perdurando el interés monopolista, llega el momento en que esa comunidad u otra en la que pueden influir establece un ordenamiento que crea monopolios para limitar la competencia [...] De esta manera, de la comunidad de interesados nace una "comunidad jurídica»: los interesados son «miembros compañeros de una comunidad jurídica». Este proceso de "cierre» de una comunidad, como lo llamaremos, es un proceso que se repite típicamente y constituye la raíz de la "propiedad» territorial, así como de todos los gremios y demás grupos monopolistas» (1993: 276) ${ }^{21}$.

Los tratamientos teóricos del cierre social en relación con el idioma se han centrado en el cierre de las elites con el propósito de lograr y conservar el poder (Myers-Scotton, 1982, 1993; Pool, 1993) 22. Así, en el plano empíri-

20. Indicaremos aquí, simplemente, que una norma es «una descripción verbal de una conducta tal que muchos de los miembros encuentran valioso que su propia conducta y la de los demás se ajuste a ella» (Homans, 1999: 302).

21. La "propiedad territorial» es un buen ejemplo de cómo atributos de los individuos se extrapolan a lindes territoriales en señal de dominación, particularmente en el caso de los estados. La lengua puede llegar a constituir la base de la ciudadanía (Estonia), siendo la ciudadanía requisito para el acceso a los recursos públicos de un determinado estado, o bien un mecanismo discriminador en el acceso a los puestos de trabajo (Quebec) dispuesto en base a una norma sobre uso lingüístico.

22. «El cierre de las elites es un tipo de movilización social estratégica, por medio de la cual individuos en el poder establecen o mantienen sus privilegios para limitar el acceso al poder y a la mejora socioeconómica a los no miembros de la elite o a la oposición política. El cierre de las elites es posible gracias a tres proposiciones sociolingüísticas universales: (1) no todos los individuos de la misma comunidad hablan las mismas variedades lingüísticas; (2) las diferentes variedades en uso en una comunidad tiene diferentes usos situacionales; (3) todas las variedades son positiva o negativamente evaluadas por los miembros de la comunidad de acuerdo con un tipo específico de interacción» (Myers-Scotton, 1993: 149). 
co, Myers-Scotton se ha centrado en el estudio de estados africanos donde la oficialidad de la lengua inglesa se ha mostrado como una estrategia segmentadora de los individuos ${ }^{23}$, mientras que Pool ha desarrollado formalmente dicho modelo de cierre lingüístico de las elites. Estos enfoques se orientan hacia las clases políticas dirigentes como grupos relativamente reducidos con respecto a la población total. No se trata de un planteamiento orientado a la legitimación de las elites y el sistema político, sino de un mecanismo orientado a limitar el acceso a los puestos de privilegio a la población gobernada. Por otra parte, siguiendo a Myers-Scotton, la segmentación lingüística se hace posible en sociedades con escasa movilidad social. La integración socioeconómica se caracteriza por tener una forma gradual, en lugar de rígida, en la curva socioeconómica que ubica a grupos separados en una misma nación. La posición en la curva define el control de cada grupo sobre los recursos socioeconómicos de la nación. Con una forma gradual de la curva socioeconómica la movilidad es posible, esto es, grupos enteros o individuos poseen el potencial para cambiar su posición socioeconómica. En definitiva, para que los individuos deseen añadir un idioma a su repertorio, deben estar $(a)$ insatisfechos con su estatus socioeconómico presente $(b)$ confiados de que añadir una lengua a su repertorio mejorará su estatus socioeconómico (MyersScotton, 1982: 85). Es por este último motivo (b) que existen dos tipos de inmigrantes con diferentes actitudes y preferencias hacia la lengua: (1) los que aprenden la que pueden y les resulta más útil en el lugar de destino y (2) los que emigran porque ya han aprendido la lengua más útil. Los incentivos para la capitalización lingüística dependen de la capacidad de integración socioeconómica de la sociedad ${ }^{24}$.

\section{Conclusiones}

Los beneficios del intercambio generan la necesidad de establecer pautas sobre cómo realizarlos, entre las que se encuentran una estandarización de los idiomas y la moneda. Ello supone la fácil adhesión de más individuos al intercambio, produciéndose un aumento de los círculos o comunidades de intercambio. Por otra parte, el aumento del círculo supone diferenciación funcional de los miembros e individualización, generándose expectativas particulares sobre los recursos. En particular, el aumento del círculo económico

23. «En la medida que diferentes personas poseen variedades diferentes, en la medida que la misma variedad no es apropiada para toda situación, y dado que las variedades son evaluadas diferencialmente, por todo ello, el cierre de las elites está disponible como una poderosa estrategia lingüística» (Myers-Scotton, 1993: 150-51).

24. Por otra parte, cabe destacar la relación entre el cierre social y determinadas formas de discriminación lingüística que conducen de facto a formas de segregación entre grupos lingüísticos donde la discriminación individual en los salarios conduce al resultado social de una discriminación en firmas segregadas, así como a una estratificación vertical en función de la lengua (Becker, 1971; Lang, 1993). 
puede implicar alejamiento de los recursos, es decir, aumento de la distancia, no controlada, entre el individuo y los recursos que se intercambian en una comunidad. Las transformaciones en las pautas de intercambio económico operan sobre las actitudes individuales de los agentes, persiguiendo su bienestar por medio de sus decisiones sobre el idioma. Es por ello, que emergen determinadas normas de interacción que limitan estratégicamente el tamaño de los círculos de intercambio, como determinadas pautas de interacción lingüística que pretenden segmentar lingüísticamente el acceso a los recursos. El hecho de que las comunidades de intercambio lingüístico no coincidan con las comunidades monetarias, no necesariamente indica, en el momento presente, un desinterés por el intercambio económico internacional, sino más bien la posibilidad de extraer mayor utilidad a los recursos presentes en un determinado marco idiomático de intercambio, es decir, la capacidad para agrupar a individuos en torno a intereses comunes, intereses aún alejados de un auténtico y definitivo círculo económico de carácter global.

Ritzer indica sobre Simmel que éste "parece tener una perturbadora tendencia a reducir las estructuras sociales a no mucho más que pautas de interacción $[s i c] »$. A mi modo de ver, son precisamente esas pautas de interacción las que rigen los "hechos sociales» - proporcionando incentivos y sanciones. Por tanto, difiero con Ritzer en que «en el nivel macro, Simmel no nos ofreció gran cosa sobre las estructuras sociales» (Ritzer, 1995: 331), pues al contrario, junto a los desarrollos sobre la racionalidad individual propuestos por Weber, Simmel permite la integración de los aspectos micro y macro de la explicación sociológica, integrando las formas de interacción como mecanismos de transformación de las estructuras y los sujetos.

\section{Bibliografía}

BECKer, G. (1971). The economics of discrimination. Chicago: The University of Chicago Press.

BLAU, P.M. (1982). Intercambio y poder en la vida social. Barcelona: Hora.

CARR, J. (1985). «Le bilingüisme au Canada: l'usage consacre-t-il l'anglais monopole naturel?» en VaillancourT, F. (ed.): Economie et langue. Québec: Documenta tion du Conseil de la langue française, 20: 27-37.

Coleman, J.S. (1990). Foundations of Social Theory. Cambridge: The Belknap Press of Harvard University Press.

CHISWICK, B.R.; MiLleR, P.W. (1995). «The Endogeneity between Language and Earnings: International Analyses». En: Journal of Labor Economics, 13: 246-288.

Church, J.; KING, I. (1993). Bilingualism and network externalities». En: Canadian Journal of Economics, 26: 337-345.

De SWAAN, A. (1998). «A Political Sociology of the World Language System (1): The Dinamics of Language Spread». En: Language Problems and Language Planing, 22: 63-75.

Fishman, J. (1979). Sociología del lenguaje. Madrid: Cátedra. 
Friedman, D. (1977). «A Theory of the Size and Shape of Nations». En: Journal of Political Economy, 85: 59-77.

Gellner, E (1988). Naciones y nacionalismo. Madrid: Alianza Universidad.

GRIN, F. (1990). «The economic aproach to Minority Languages». En: Journal of Mul tilingual and Multicultural Development, 11: 153-174.

Laitin, D.D. (1988). «Language games». En: Comparative Politics, 9: 228-302.

- (1992). «Language Normalization in Estonia and Catalonia». En: JBS, 23: 149-165.

- (1989). "Linguistic Revival: Politics and Culture in Catalonia». En: Society for Comparative Study of Society and History, 12: 297-317.

Laitin, D.D.; RodrígueZ, G. (1992). «Language, Ideology, and the Press in Catalonia». En: American Anthropologist, 94: 9-30.

LAITIN, D.D.; SOlÉ, C.; KalYVAS, S.N. (1994). «Language and the Construction for States: The Case of Catolonia in Spain». En: Politics \& Society, 22: 5-29.

LAPONCE, J.A. (1985). «Language and communication: the rise of the monolingual state». En: Cioffi-Revilla, R.L.; Merrit, R.; Zinnes, D.A. (eds.). Communi cation and interaction in global politics. Beverly Hills: SAGE.

- (1993). «Do languages behave like animals?». En: International Journal of the Sociology of Language, 103: 19-30. México: Fondo de Cultura Económica.

MyeRs SCOTTON, C. (1982). «Learning Lingua Francas and Socioeconomic Integration: Evidence from Africa». En: CoOpER, R.L. (ed.). Language Spread: Studies in Difusion and Social Change. Bloomington: Indiana University Press, 63-94.

- (1993). «Elite closure as a powerful language strategy: The African case». En: International Journal of the Sociology of Language, 103: 149-163.

Olson, M. (1992). La lógica de la acción colectiva: bienes públicos y la teoría de grupos. México, D.F: Limusa.

Pool, J. (1991). "The World Language Problem». En: Rationality and Society, 3: 78-105.

- (1993). «Linguistic Exploitation». En: International Journal of the Sociology of Lan guage, 103: 31-55.

REQUENA, F. (1998). «Grupos abiertos/cerrados». En: Giner, S.; LAMO DE ESPINOSA, E.; Torres, C. (eds.). Diccionario de Sociología. Madrid: Alianza Editorial, 339.

Ritzer, G. (1995). Teoría sociológica contemporánea. Madrid: McGraw-Hill.

Simmel, G. (1977). Filosofia del dinero. Madrid: Instituto de Estudios Políticos.

- (1986). Sociología: estudios sobre las formas de socialización. Vol. 2. Madrid: Alianza.

Smith, A.D. (1976). Las teorías del nacionalismo. Barcelona: Península.

TiveY, L. (1987). «Estados naciones y economía». En: TiveY, L. (ed.), Estado Nación. Barcelona: Península: 79-105.

Weber, M. (1981). «La sociología de Simmel». En: Papers: Revista de Sociología, 15.

- (1993). Economía y sociedad: esbozo de sociología comprensiva. México: Fondo de Cultura Económica. 